\title{
LARGE-SCALE FILAMENTARY STRUCTURES AROUND THE VIRGO CLUSTER REVISITED
}

\author{
Suk Kim ${ }^{1,2}$, Soo-Chang Rey ${ }^{1,7}$, Martin Bureau ${ }^{3}$, Hyein Yoon $^{4}$, Aeree Chung ${ }^{4}$, Helmut Jerjen ${ }^{5}$, Thorsten Lisker ${ }^{6}$, \\ Hyunjin Jeong ${ }^{2}$, Eon-Chang Sung ${ }^{2}$, Youngdae Lee ${ }^{1}$, Woong LeE ${ }^{1}$, and Jiwon Chung ${ }^{1}$ \\ ${ }^{1}$ Department of Astronomy and Space Science, Chungnam National University, 99 Daehak-ro, Daejeon 305-764, Korea; screy@cnu.ac.kr \\ ${ }^{2}$ Korea Astronomy \& Space Science institute, 776 Daedeokdae-ro, Daejeon 305-348, Korea; star4citizen@kasi.re.kr \\ ${ }^{3}$ Sub-department of Astrophysics, Department of Physics, University of Oxford, Denys Wilkinson Building, Keble Road, Oxford OX1 3RH, UK \\ ${ }^{4}$ Department of Astronomy and Yonsei University Observatory, Yonsei University, Seoul 120-749, Korea \\ ${ }^{5}$ Research School of Astronomy and Astrophysics, The Australian National University, Cotter Road, Weston, ACT 2611, Australia \\ ${ }^{6}$ Astronomisches Rechen-Institut, Zentrum für Astronomie der Universität Heidelberg (ZAH), Mönchhofstraße 12-14, D-69120 Heidelberg, Germany \\ Received 2016 September 7; revised 2016 October 21; accepted 2016 October 26; published 2016 December 19
}

\begin{abstract}
We revisit the filamentary structures of galaxies around the Virgo cluster, exploiting a larger data set, based on the HyperLeda database, than previous studies. In particular, this includes a large number of low-luminosity galaxies, resulting in better sampled individual structures. We confirm seven known structures in the distance range $4 h^{-1} \mathrm{Mpc}<\mathrm{SGY}<16 h^{-1} \mathrm{Mpc}$, now identified as filaments, where SGY is the axis of the supergalactic coordinate system roughly along the line of sight. The Hubble diagram of the filament galaxies suggests they are infalling toward the main body of the Virgo cluster. We propose that the collinear distribution of giant elliptical galaxies along the fundamental axis of the Virgo cluster is smoothly connected to two of these filaments (Leo II A and B). Behind the Virgo cluster ( $16 h^{-1} \mathrm{Mpc}<\mathrm{SGY}<27 h^{-1} \mathrm{Mpc}$ ), we also identify a new filament elongated toward the NGC 5353/4 group ("NGC 5353/4 filament") and confirm a sheet that includes galaxies from the W and $\mathrm{M}$ clouds of the Virgo cluster ("W-M sheet"). In the Hubble diagram, the NGC 5353/4 filament galaxies show infall toward the NGC 5353/4 group, whereas the W-M sheet galaxies do not show hints of gravitational influence from the Virgo cluster. The filamentary structures identified can now be used to better understand the generic role of filaments in the build-up of galaxy clusters at $z \approx 0$.
\end{abstract}

Key words: galaxies: clusters: individual (Virgo cluster) - galaxies: dwarf - large-scale structure of universe

\section{INTRODUCTION}

The visible matter distribution of the universe forms a complex web-like network composed of filaments and sheets separated by voids (Bond et al. 1996; Aragón-Calvo et al. 2010). Galaxies are continuously funneled into higher density cluster environments through these structures. In the hierarchical structure formation scenario, $z \approx 0$ filaments are the current end point of large-scale structure evolution. Investigating filamentary structures around dynamically young clusters should thus yield valuable information on their assembly histories. Studying the large-scale galaxy distribution around the Virgo cluster, the nearest rich, young cluster (Aguerri et al. 2005), should therefore provide constraints on its formation and evolution at the current epoch.

From three-dimensional mapping of nearby galaxies, Tully (1982, hereafter T82) identified prolate and oblate overdensities of galaxies around the Virgo cluster. These were, however, not clearly revealed as conventional narrow filaments, but were rather sparse, mainly due to the limited sample size. A better characterization of these structures requires improved statistics from larger galaxy samples, particularly those with fainter galaxies.

Recent spectroscopic surveys of galaxies such as the Sloan Digital Sky Survey (SDSS; e.g., SDSS Data Release 7, Abazajian et al. 2009) do enable the exploration of possible filamentary structures within an extensive volume around the Virgo cluster. Moreover, radial velocity information on numerous galaxies combined with distance data allows us to investigate potential dynamical relationships between the

\footnotetext{
${ }^{7}$ Author to whom any correspondence should be addressed.
}

filaments and the Virgo cluster. The primary goal of our study is thus to revisit the large-scale structures in the vicinity of the Virgo cluster using an up-to-date statistically robust data set, to test whether the T82 structures can be better characterized and identified as filaments. We also search for previously unknown structures that may now be apparent. Finally, we investigate whether the structures are physically connected to the Virgo cluster using Hubble diagrams. Throughout this paper, we assume a Hubble constant of $H_{0}=100 h \mathrm{~km} \mathrm{~s}^{-1} \mathrm{Mpc}^{-1}$, where $h=0.74$ (Tully et al. 2008, hereafter T08).

\section{DATA AND ANALYSIS}

While the SDSS is large and homogeneous, it does not cover most of the southern hemisphere and the spectroscopy is incomplete for both bright $(r \lesssim 16)$ and faint $(r \gtrsim 20)$ objects (e.g., Kim et al. 2014). We therefore adopt here the HyperLeda database $^{8}$ (Paturel et al. 2003) which, while heterogeneous and necessarily incomplete, does contain multiple other surveys in addition to the SDSS Data Release 7 (Abazajian et al. 2009).

For our analysis, we extracted all galaxies with available radial velocities less than $6000 \mathrm{~km} \mathrm{~s}^{-1}$ in the region $115^{\circ}<\mathrm{R}$. A.(J2000) $<240^{\circ}$ and $-35^{\circ}<\operatorname{decl} .(\mathrm{J} 2000)<60^{\circ}$ (see Figure 1). This ensures the inclusion of all structures potentially associated with the Virgo cluster (with a mean radial velocity of $\approx 1000 \mathrm{~km} \mathrm{~s}^{-1}$ and a velocity dispersion of $\approx 800 \mathrm{~km} \mathrm{~s}^{-1}$; Binggeli et al. 1993). However, as no distinct structure in the vicinity of the Virgo cluster is identified beyond a velocity of $3300 \mathrm{~km} \mathrm{~s}^{-1}$, we henceforth only discuss the 9168 galaxies within that range. We examined the radial velocities of 755

\footnotetext{
http://leda.univ-lyon1.fr
} 


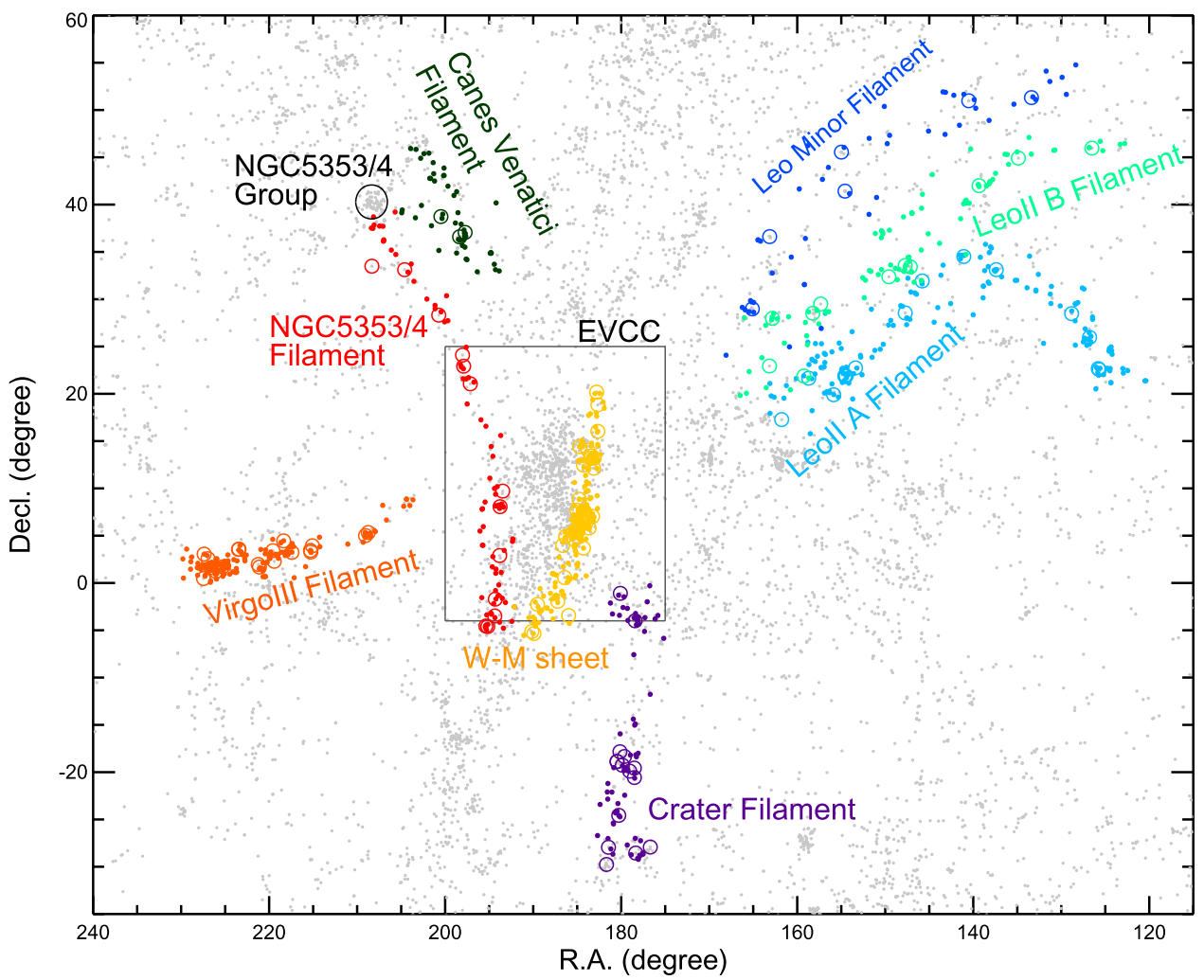

Figure 1. Spatial distribution of galaxies in seven filaments and one sheet around the Virgo cluster in the equatorial coordinate system. Bright $\left(M_{B}<-19\right)$ and faint $\left(M_{B}>-19\right)$ galaxies are denoted by large open circles and small filled circles, respectively. Different colors denote different structures. The large rectangular box is the region of the Extended Virgo Cluster Catalog (EVCC; Kim et al. 2014). The gray dots represent galaxies not associated with the Virgo cluster or a particular structure.

SDSS filament galaxies, finding a mean difference of $6 \mathrm{~km} \mathrm{~s}^{-1}$ and a dispersion of $63 \mathrm{~km} \mathrm{~s}^{-1}$ with respect to HyperLeda velocities (the latter typically averaging multiple surveys). We therefore conclude that the radial velocity information of our sample is not significantly affected by the compilation of different sources in HyperLeda.

To construct an accurate spatial distribution of the galaxies, we first converted the observed heliocentric radial velocities to velocities relative to the centroid of the Local Group, and then applied a correction for infall into the Virgo cluster (Mould et al. 2000). Based on the assumption of a linear relationship between redshift and distance, all galaxies were then mapped in the three-dimensions of the Cartesian supergalactic coordinate system: SGX, SGY, SGZ (T08). Our search for filamentary structures was conducted within the cuboid enclosed by the following limits

$$
\begin{aligned}
& -25 h^{-1} \mathrm{Mpc}<\mathrm{SGX}<25 h^{-1} \mathrm{Mpc}, \\
& 4 h^{-1} \mathrm{Mpc}<\mathrm{SGY}<27 h^{-1} \mathrm{Mpc}, \\
& -25 h^{-1} \mathrm{Mpc}<\mathrm{SGZ}<25 h^{-1} \mathrm{Mpc} .
\end{aligned}
$$

The use of this extended region enables a more accurate characterization of large-scale filamentary structures than the study of T82. We rejected galaxies within a radius of $3.6 \mathrm{Mpc}$ from the Virgo cluster center (at least two times the virial radius; McLaughlin 1999), to avoid contamination from cluster galaxies with large infall velocities that are gravitationally bound to the cluster inside the infalling region (Mamon et al. 2004; Falco et al. 2014). This leaves 8401 galaxies with which to search for large-scale structures.
The SGX-SGZ plane is the best place to look for large-scale structures, as positional errors are small in this plane (roughly in the plane of the sky) but substantial along the SGY axis (roughly along the line of sight and thus affected by deviations from the Hubble flow). The detection of filaments was thus performed by applying the following steps:

1. We constructed a series of different SGX-SGY-SGZ volume slices with an arbitrary depth of $2 h^{-1} \mathrm{Mpc}$ along the SGY axis. Candidate structures were then selected by visual inspection of the SGX-SGZ projection of each slice, looking for overdense and long (i.e., filamentary) galaxy distributions. If a candidate structure was continuously present in consecutive slices, we accordingly estimated its full range in three-dimensions.

2. We performed three-dimensional third-order polynomial fitting with weighting by the local galaxy density to the visually selected candidate structures. If the standard deviation of the fit to a candidate structure was less than $1.5 h^{-1} \mathrm{Mpc}$ (comparable to or less than the typical thickness of filaments in simulations and observations; e.g., Colberg et al. 2005; Akahori \& Ryu 2010; Choi et al. 2010; Vazza et al. 2014), the structure was retained and classified as a filament. If not, the candidate structure was rejected. We further applied a two-sigma clip to the galaxies around the fitted lines (i.e., the filament spines), to extract the galaxies that belong to each filament.

3 . The authenticity of each candidate structure was verified by looking at a multitude of SGX-SGY-SGZ projections, and the more diffuse (i.e., non-filamentary) nature of the rejected structures was assessed (chance projections, sheets, etc.). 
With the search strategy above, we identified seven filaments and one sheet in the volume surrounding the Virgo cluster, divided into two subsamples with distinct SGY ranges (see Section 3). Figure 1 shows the spatial distribution of the 1013 galaxies belonging to these structures in the equatorial coordinate system. The properties of these structures are summarized in Table 1 . We note that all structures mostly consist of faint galaxies $\left(M_{B}>-19 ; \approx 88 \%\right.$ of the total sample).

Finally, we collected redshift-independent distances of sample galaxies from the NASA/IPAC Extragalactic Database $^{9}$ (NED), with 229 matches. We calculated the distances $\left(R_{\mathrm{VC}}\right)$ and radial velocities $\left(V_{\mathrm{VC}}\right)$ of these galaxies relative to the Virgo cluster center (Karachentsev \& Nasonova 2010), adopting a distance of the Virgo cluster from us of $16.5 \mathrm{Mpc}$ (Mei et al. 2007). In this instance, for the calculation of $V_{\mathrm{VC}}$ (T08), the heliocentric radial velocities were corrected for the motion of the local sheet and local void only (and not for Virgo-centric infall). Finally, peculiar velocities $\left(\mathrm{PV}_{\mathrm{VC}}\right)$ were derived using the following equation:

$$
\mathrm{PV}_{\mathrm{VC}}=V_{\mathrm{VC}}-R_{\mathrm{VC}} \times H_{0} .
$$

\section{RESULT}

\subsection{Virgo-related Structures $\left(4 h^{-1} \mathrm{Mpc}<\mathrm{SGY}<16 h^{-1} \mathrm{Mpc}\right)$}

Figure 2 presents the distribution of six filaments with $4 h^{-1} \mathrm{Mpc}<\mathrm{SGY}<16 h^{-1} \mathrm{Mpc}$ in the SGX-SGZ projected plane (Figure 2(a)) and the SGX-SGY-SGZ three-dimensional space (Figure 2(b)). We named these filaments after the structures designated as clouds or spurs by T82 (Figure 2(c)). By construction, all the filaments are narrower and longer than the broader and more diffuse T82 structures. All are also elongated toward the Virgo cluster, suggesting they are related to it.

It is interesting to note that when we apply our search strategy to the T82 sample, the T82 structures are not identified as filaments. This is most likely due to the small T82 sample size, as the number of galaxies in each filament is two or three times larger in our sample.

In addition, while the majority of galaxies in T82 are bright $\left(M_{B}<-19\right)$, our structures are mainly composed of less luminous galaxies $\left(M_{B}>-19\right)$. Bright galaxies (large open circles in Figures 1-4) exhibit an uneven distribution with numerous gaps along the filaments. Conversely, the large number of low-luminosity galaxies available here (small filled circles in Figures 1-4) helps to better delineate continuous filaments by filling in the gaps.

The Leo II cloud appears to be a multi-stem clump rather than a single prolate one (Figure 2(c)). Indeed, in a more extensive area around the Leo II cloud, we identified two filaments, Leo II A and B. The Leo II B filament traces a sparse region of the cloud, whereas the Leo II A filament includes the main clump of the Leo II cloud and is one of the largest structures we detect $\left(16 h^{-1} \mathrm{Mpc}\right.$ long in the SGX-SGZ plane), with a curved shape. It is known that warped or irregular filaments are more common and are on average longer than straight filaments (Colberg et al. 2005).

Over 150 galaxies in the 6 filaments identified in Figures 2(a) and (b) have a redshift-independent distance.

\footnotetext{
https://ned.ipac.caltech.edu
}

Their Hubble diagram in the Virgo-centric reference frame is presented in Figure 2(d). Most galaxies in all these filaments exhibit a distinct deviation of their radial velocities from an unperturbed Hubble flow (red line; see also the median values in the inset), suggesting appreciable infall toward the Virgo cluster. We also plot a model of the radial infall velocity profile of the Virgo cluster (blue line), adopting the model of Falco et al. (2014; Equation (6), representing the mean velocity of infalling galaxies in the outer regions of the cluster) with a virial radius of $1.55 \mathrm{Mpc}$ (McLaughlin 1999) and a virial velocity of $800 \mathrm{~km} \mathrm{~s}^{-1}$ (Binggeli et al. 1993). The observed distribution of radial velocities of the filament galaxies is entirely consistent with the expected infall profile, confirming that all these filaments are dynamically connected to the Virgo cluster (see also Karachentsev et al. 2014).

\subsection{Structures behind the Virgo Cluster $\left(16 h^{-1} \mathrm{Mpc}<\mathrm{SGY}<27 h^{-1} \mathrm{Mpc}\right)$}

Figures 3(a) and (b) are analogous to Figures 2(a) and (b) but for $16 h^{-1} \mathrm{Mpc}<\mathrm{SGY}<27 h^{-1} \mathrm{Mpc}$. They show one filament and one sheet identified behind the Virgo cluster, at mean distances of 33 and $41 \mathrm{Mpc}$ from us, respectively.

The filament (red circles), which we call the NGC 5353/4 filament, is long and thin and extends out from the NGC 5353/ 4 group, running tangentially past the Virgo cluster rather than pointing toward it. Filaments running from the vicinity of the NGC 5353/4 group to the Virgo (e.g., Canes Venatici filament, see the gray rectangle in Figure 3(a)) and Coma clusters in different SGY ranges have been reported in the past (Tully \& Trentham 2008; Pomarède et al. 2015), but the NGC 5353/ 4 filament identified here is not specifically referred to in previous studies.

17 galaxies in the NGC 5353/4 filament have a redshiftindependent distance. Their Hubble diagram in the NGC 5353/ 4 group-centric reference frame is presented in Figure 3(c). All but one NGC 5353/4 filament galaxies show a clear negative offset from the Hubble flow, with a mean of $-242 \mathrm{~km} \mathrm{~s}^{-1}$, indicating that they are infalling toward the NGC 5353/4 group. Recently, Pomarède et al. (2015) identified the Arrowhead mini-supercluster, in which the NGC 5353/4 group is located at the edge of the Arrowhead flow pattern in the velocity field. They also suggested that the NGC 5353/4 group is located at the boundary of the flow pattern of the Laniakea supercluster (Tully et al. 2014). The NGC 5353/4 filament thus appears to be a small structure bridging the Arrowhead and Laniakea superclusters (see arrows in Figure 3(a) for the directions to the two superclusters).

A thin sheet structure (yellow circles) is also clearly visible in Figures 3(a) and (b). While it appears filament-like in the projected plane (Figure 3(a)), it is clearly flattened (Figure 3(b)), with an axial ratio SGX:SGY:SGZ = 9:6:1. Since this structure includes the $\mathrm{W}$ and $\mathrm{M}$ clouds as well as the western part of the southern extension of the Virgo cluster, we name it the W-M sheet. The existence of this sheet was however alluded to by Binggeli et al. (1993), and a connection between the $\mathrm{W}$ and $\mathrm{M}$ clouds was also suggested (e.g., Ftaclas et al. 1984; Yoon et al. 2012).

54 galaxies in the $\mathrm{W}-\mathrm{M}$ sheet have a redshift-independent distance. Their Hubble diagram in the Virgo-centric reference frame is presented in Figure 3(d). The W-M sheet galaxies have a large scatter around the Hubble flow with no trend, 
Table 1

Filamentary Structures around the Virgo Cluster

\begin{tabular}{|c|c|c|c|c|c|c|c|c|c|c|c|c|}
\hline \multirow[b]{2}{*}{ Name } & \multirow[b]{2}{*}{$\begin{array}{c}\text { SGX } \\
\left(h^{-1} \mathrm{Mpc}\right)\end{array}$} & \multirow[b]{2}{*}{$\begin{array}{c}\text { SGY } \\
\left(h^{-1} \mathrm{Mpc}\right)\end{array}$} & \multirow[b]{2}{*}{$\begin{array}{c}\text { SGZ } \\
\left(h^{-1} \mathrm{Mpc}\right)\end{array}$} & \multirow[b]{2}{*}{$\begin{array}{c}\left.\mathrm{cz}^{-1}\right) \\
\left(\mathrm{km} \mathrm{s}^{-1}\right.\end{array}$} & \multirow[b]{2}{*}{$\begin{array}{c}\text { Length } \\
\left(h^{-1} \mathrm{Mpc}\right)\end{array}$} & \multirow[b]{2}{*}{$\begin{array}{l}R_{\mathrm{VC}} \\
(\mathrm{Mpc})\end{array}$} & \multirow[b]{2}{*}{$\begin{array}{l}\text { Peculiar Velocity } \\
\left(\mathrm{km} \mathrm{s}^{-1}\right)\end{array}$} & \multirow[b]{2}{*}{$\begin{array}{l}\text { Distance }_{\mathrm{MW}} \\
(\mathrm{Mpc})\end{array}$} & \multicolumn{2}{|c|}{ This Work } & \multicolumn{2}{|c|}{ Tully (1982) } \\
\hline & & & & & & & & & $N$ & $N_{\text {faint }}$ & $N$ & $N_{\text {faint }}$ \\
\hline (1) & (2) & (3) & (4) & (5) & (6) & (7) & (8) & (9) & (10) & (11) & (12) & (13) \\
\hline Leo II A & $0.21 \sim 10.36$ & $9.26 \sim 15.05$ & $-15.47 \sim-4.16$ & $1171 \sim 2267$ & 16.0 & $11.71 \sim 46.68$ & $-213.98(283.02)$ & $26.30(4.86)$ & 180 & 165 & $97^{\mathrm{b}}$ & $45^{\mathrm{b}}$ \\
\hline Leo II B & $0.30 \sim 15.65$ & $10.90 \sim 14.56$ & $-9.88 \sim-3.51$ & $1257 \sim 2267$ & 15.5 & $10.84 \sim 32.19$ & $-282.04(306.53)$ & $26.40(4.71)$ & 105 & 94 & & \\
\hline Leo Minor & $0.55 \sim 5.89$ & $4.11 \sim 6.49$ & $-2.61 \sim-0.99$ & $505 \sim 772$ & 5.4 & $7.27 \sim 17.41$ & $-250.09(137.90)$ & $14.07(3.65)$ & 54 & 48 & 46 & 31 \\
\hline Canes Venatici & $0.78 \sim 4.37$ & $6.88 \sim 13.92$ & $1.38 \sim 4.80$ & $674 \sim 1446$ & 4.8 & $7.15 \sim 27.05$ & $-254.95(356.27)$ & $20.96(6.83)$ & 51 & 48 & 18 & 14 \\
\hline Virgo III & $-10.56 \sim-3.91$ & $9.50 \sim 15.57$ & $2.35 \sim 11.72$ & $1160 \sim 2196$ & 11.4 & $7.26 \sim 32.07$ & $-102.24(364.43)$ & $26.70(6.00)$ & 181 & 162 & 61 & 20 \\
\hline Crater & $-12.25 \sim-4.62$ & $8.36 \sim 12.70$ & $-5.91 \sim-2.98$ & $1436 \sim 1903$ & 7.9 & $8.28 \sim 19.13$ & $-148.95(138.52)$ & $23.31(3.55)$ & 84 & 69 & 35 & 6 \\
\hline NGC 5353/4 & $-16.04 \sim 4.23$ & $21.71 \sim 26.53$ & $-1.19 \sim 8.92$ & $2268 \sim 3238$ & 21.9 & $6.70 \sim 32.27$ & $-242.46^{\mathrm{a}}(306.19)$ & $41.05(7.79)$ & 102 & 89 & $\ldots$ & $\ldots$ \\
\hline W-M sheet & $-13.38 \sim-1.66$ & $16.03 \sim 24.99$ & $-3.10 \sim-1.10$ & $1806 \sim 2968$ & 11.9 & $1.45 \sim 65.64$ & $-108.85(786.05)$ & $32.60(10.91)$ & 256 & 221 & $\ldots$ & $\ldots$ \\
\hline
\end{tabular}

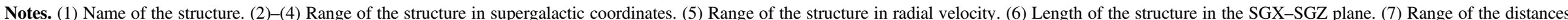

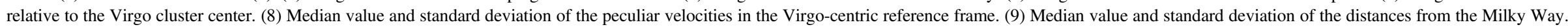
(10)-(11) Number of total and faint $\left(M_{B}>-19\right)$ galaxies. (12)-(13) Number of total and faint $\left(M_{B}>-19\right)$ galaxies in Tully (1982).

${ }^{a}$ Median peculiar velocity and standard deviation in the NGC 5353/4 group-centric reference frame.

${ }^{\mathrm{b}}$ Tully (1982) designated the Leo II cloud as a single structure. 

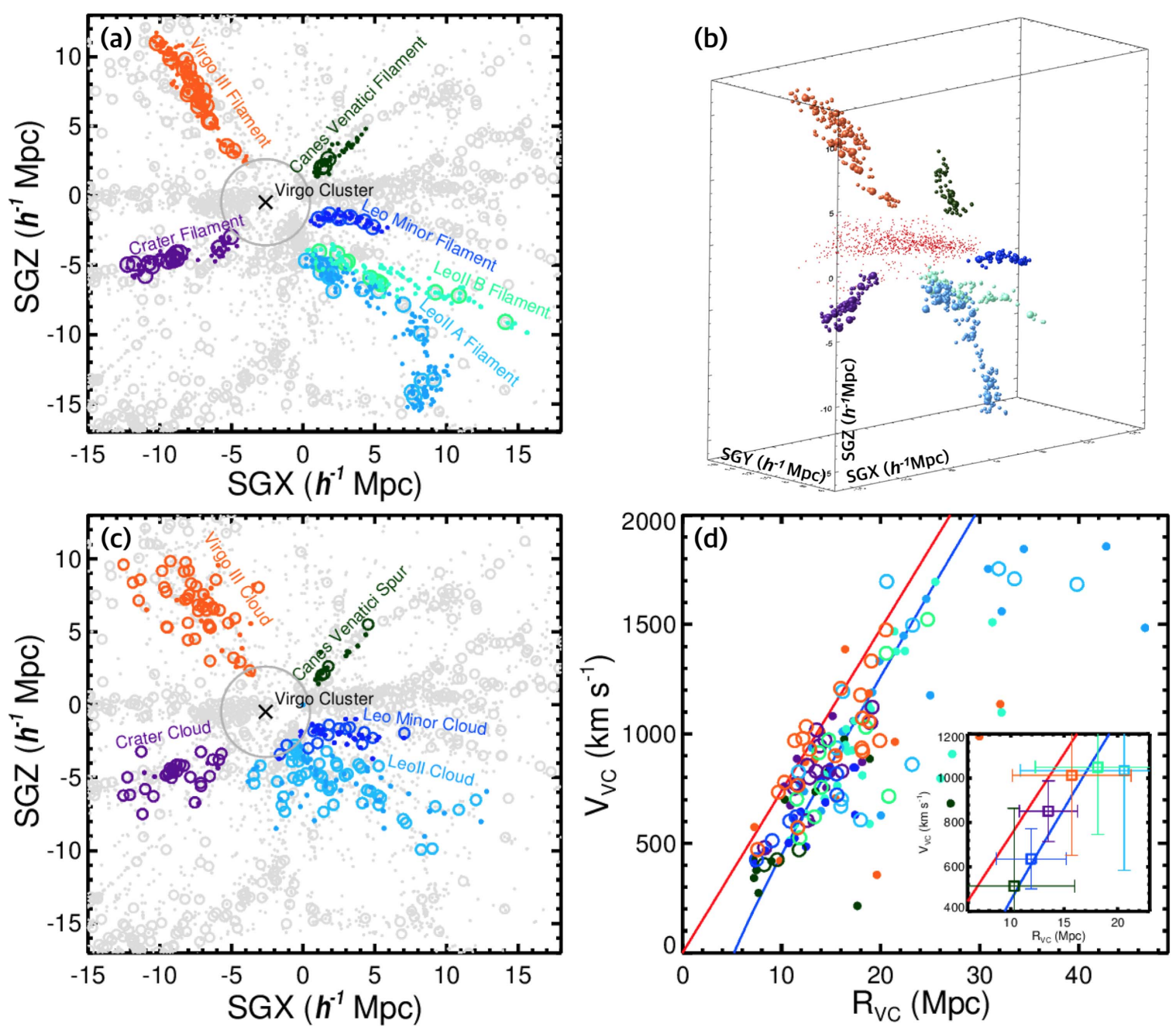

Figure 2. Spatial distribution (a)-(c) and Hubble diagram (d) of six filaments in the range $4 h^{-1} \mathrm{Mpc}<\mathrm{SGY}<16 h^{-1} \mathrm{Mpc}$. Symbols are the same as in Figure 1. (a) Projected spatial distribution of the filaments in the SGX-SGZ plane. The large gray circle marks two virial radii around the Virgo cluster. (b) Three-dimensional distribution of the filaments. The red dots are Virgo cluster galaxies in the EVCC. (c) Same as (a) for the structures mentioned by Tully (1982). (d) Hubble diagram of the filament galaxies in the Virgo-centric reference frame. The red and blue lines indicate the Hubble flow and a model of the radial infall velocity profile caused by the gravitational pull of the Virgo cluster, respectively. The inset shows the median Virgo-centric radial velocity and distance of each filament (error bars indicate one standard deviation).

indicating that the sheet is unlikely to be dynamically connected to the Virgo cluster.

\section{DISCUSSION AND CONCLUSIONS}

Cosmological numerical simulations suggest that galaxy clusters have non-spherical shapes, owing to the preferential infall of matter along the "cosmic web" (e.g., Limousin et al. 2013 and references therein). Dynamically young clusters within filaments are also expected to be more elongated than relaxed clusters. Moreover, it has been noted that the Virgo cluster exhibits a noticeably non-spherical shape, elongated along a direction almost parallel to the line of sight, albeit primarily when considering galaxies near the cluster center (e.g., Binggeli et al. 1987; West \& Blakeslee 2000; Mei et al. 2007).

In Figure 4(a), we show the spatial distribution of galaxies in the Virgo cluster using only galaxies classified as "certain" cluster members in the Extended Virgo Cluster Catalog (EVCC, Kim et al. 2014, black open circles inside a large rectangular box). Using HyperLeda, we also plot the galaxies (gray open circles) within the cluster zero-velocity surface (large gray circle with a $26^{\circ}$ radius; Karachentsev \& Nasonova
2010). Noticeably, the bright elliptical galaxies $\left(M_{B}<-19\right.$; red open circles) are all aligned. The galaxies in the western outskirts of the cluster (i.e., $180^{\circ}<$ R.A. $<160^{\circ}$ ) also exhibit a filamentary distribution, with a major axis similar to that of the bright ellipticals nearer the cluster center. Moreover, this collinear distribution of cluster galaxies appears to be smoothly connected to the Leo II A and B filaments identified here (cyan and green circles).

The genuine alignment of the galaxies in three-dimensions is confirmed by the distribution of the galaxies with available redshift-independent distances in the plane of R.A. versus distance from us (Figure 4(b)). Instead of a random distribution, the galaxy distances are systematically increasing from the cluster center to the filaments. This systematic trend supports the suggestion that the galaxy distribution along the fundamental axis of the Virgo cluster is associated with the Leo II A and B filaments. West \& Blakeslee (2000) claimed that the collinear distribution of the brightest ellipticals near the cluster center may be part of a filament connecting the Virgo cluster to Abell 1367 (see the arrow indicating the direction to Abell 1367 in Figure 4(b)), but our data suggest that a 

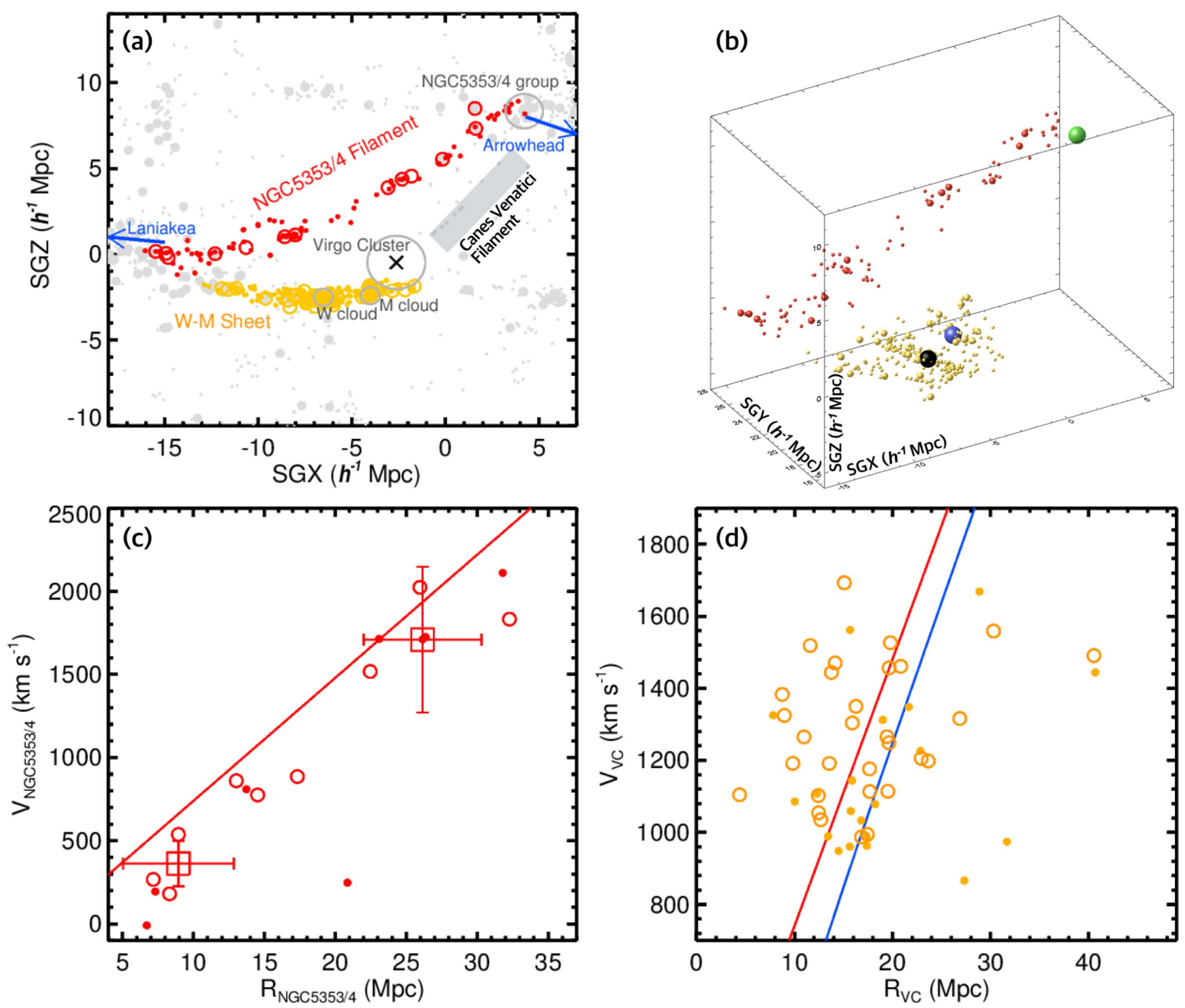

Figure 3. Spatial distribution (a)-(b) and Hubble diagram (c)-(d) of the NGC 5353/4 filament (red circles) and the W-M sheet (yellow circles) in the range $16 h^{-1} \mathrm{Mpc}<\mathrm{SGY}<27 h^{-1}$ Mpc. Symbols are the same as in Figure 1. (a) Projected spatial distribution of the structures in the SGX-SGZ plane. The gray rectangle marks the region of the Canes Venatici filament. The directions to the Arrowhead and Laniakea superclusters are indicated with blue arrows (Tully et al. 2014; Pomarède et al. 2015). (b) Three-dimensional distribution of the structures. The black, blue, and green spheres denote the W cloud, M cloud, and NGC 5353/4 group, respectively. (c) Hubble diagram of the NGC 5353/4 filament galaxies in the NGC 5353/4 group-centric reference frame. The red line indicates the Hubble flow, while the open squares denote the median NGC 5353/4 group-centric radial velocity and distance of galaxies in different distance ranges (error bars indicate one standard deviation). (d) Hubble diagram of the W-M sheet galaxies in the Virgo-centric reference frame. The red and blue lines indicate the Hubble flow and a model of the radial infall velocity profile caused by the gravitational pull of the Virgo cluster, respectively.

connection to the Leo II A and B filaments is more likely. In any case, our results are clear observational evidence that the spatial distribution of cluster galaxies is not completely spherically symmetric, reflecting the anisotropy of their accretions along filaments (e.g., Joachimi et al. 2015 and references therein; see also Lee et al. 2014). In this respect, comparative studies of galaxies in Virgo-related filaments with ones in the cluster itself should help to understand the growth and evolution of the cluster (S. Kim 2016, in preparation).

Filament galaxies are a crucial tool to measure galaxy cluster and group parameters. We have already estimated a new Virgo cluster dynamical mass by applying the novel method of Falco et al. (2014), based on the universal radial velocity profile of filament galaxies, to the Leo II A and Virgo III filaments (Lee et al. 2015a). We have also determined the turn-around radius of the NGC 5353/4 group by applying the Falco et al. methodology to the NGC 5353/4 filament (Lee et al. 2015b). Accurate measurements of the physical parameters of nearby galaxy clusters and groups using filaments in their vicinity can also provide independent constraints on cosmological models (see Lee et al. 2015a, 2015b for details).
Finally, filaments are prime targets for detailed investigations of the physical processes controlling the transition of field galaxies to cluster galaxies (e.g., Balogh et al. 2004; Ebeling et al. 2004; Porter et al. 2008; Yoon et al. 2012; Guo et al. 2015; Martínez et al. 2016). Indeed, as the majority of filament galaxies are faint dwarf galaxies, with low binding energies, they are easily affected by even weak perturbations. They are thus well-suited to probing the details of the multiple mechanisms (e.g., gas stripping and tidal interactions) that can affect galaxies in low-density environments before they enter higher density regions ("pre-processing"; Fujita 2004; Cybulski et al. 2014). To directly probe environmental effects in filaments, we are thus currently examining the H I morphology and kinematics of carefully selected late-type galaxies in filaments around the Virgo cluster (e.g., Yoon et al. 2015; H. Yoon 2016, in preparation). In a forthcoming paper, we will also compare dwarf galaxies in filaments with those in other environments, including cluster (e.g., Kim et al. 2014), group (e.g., Pak et al. 2014), and the field (S. Kim 2016, in preparation). 


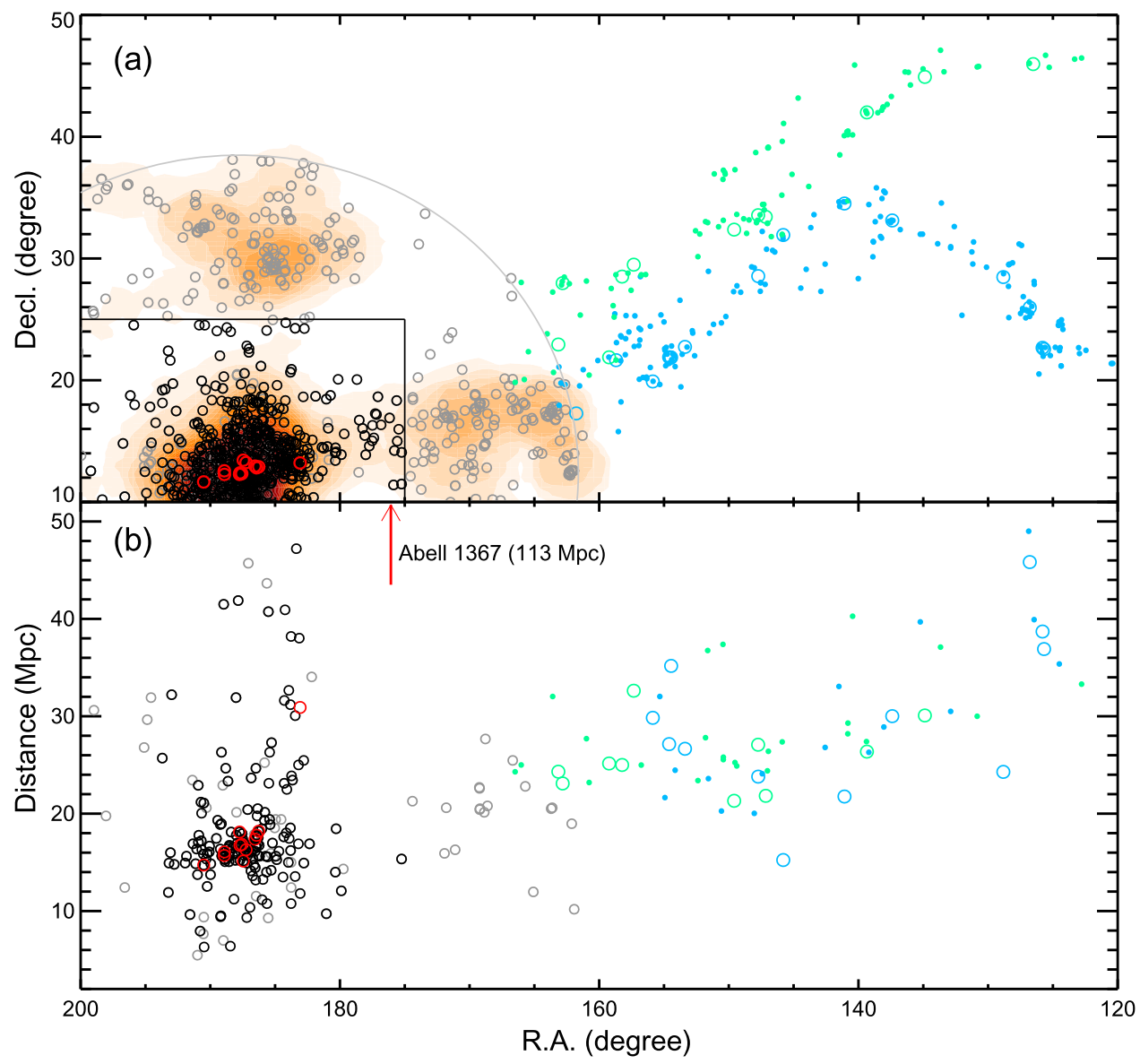

Figure 4. (a) Spatial distribution of galaxies in the Leo II A (cyan circles) and B (green circles) filaments. Red and black open circles show bright elliptical galaxies $\left(M_{B}<-19\right)$ and "certain" Virgo cluster member galaxies in the region of the EVCC (large rectangular box), respectively. The gray open circles show galaxies in the range $4 h^{-1} \mathrm{Mpc}<\mathrm{SGY}<16 h^{-1} \mathrm{Mpc}$ from HyperLeda and orange/red contours denote the local number density distribution of these galaxies. The large gray circle is the upper limit of the zero-velocity surface ( $26^{\circ}$ radius). (b) Same as (a) but in the plane of R.A. vs. distance from us. The direction to the Abell 1367 cluster and its distance from us are indicated by the annotated red arrow.

We thank the anonymous referee for the clarifications and comments that helped to improve the original manuscript. We thank Donsu Ryu and Jounghun Lee for helpful discussions. This research was supported by the Basic Science Research Program through the National Research Foundation of Korea (NRF) funded by the Ministry of Education, Science, and Technology (2015R1A2A2A01006828). Support was also provided by the NRF of Korea to the Center for Galaxy Evolution Research (No. 2010-0027910). S. Kim acknowledges support from a National Junior Research Fellowship of NRF (No. 2011-0012618). H. Jeong acknowledges support from the NRF funded by the Ministry of Education (NRF2013R1A6A3A04064993). This study was also financially supported by the research fund of Chungnam National University in 2014. This work has been also supported by Science Fellowship of POSCO TJ Park Foundation and the NRF grant No. 2015R1D1A1A01060516. H. Jerjen acknowledges the support of the Australian Research Council through Discovery Project DP150100862.

\section{REFERENCES}

Abazajian, K. N., Adelman-McCarthy, J. K., Agüeros, M. A., et al. 2009, ApJS, 182, 543

Aguerri, J. A. L., Gerhard, O. E., Arnaboldi, M., et al. 2005, AJ, 129, 2585

Akahori, T., \& Ryu, D. 2010, ApJ, 723, 476
Aragón-Calvo, M. A., van de Weygaert, R., \& Jones, B. J. T. 2010, MNRAS, 408, 2163

Balogh, M., Eke, V., Miller, C., et al. 2004, MNRAS, 348, 1355

Binggeli, B., Popescu, C. C., \& Tammann, G. A. 1993, A\&AS, 98, 275

Binggeli, B., Tammann, G. A., \& Sandage, A. 1987, AJ, 94, 251

Bond, J. R., Kofman, L., \& Pogosyan, D. 1996, Natur, 380, 603

Choi, E., Bond, N. A., Strauss, M. A., et al. 2010, MNRAS, 406, 320

Colberg, J. M., Krughoff, K. S., \& Connolly, A. J. 2005, MNRAS, 359, 272

Cybulski, R., Yun, M. S., Fazio, G. G., \& Gutermuth, R. A. 2014, MNRAS, 439, 3564

Ebeling, H., Barrett, E., \& Donovan, D. 2004, ApJL, 609, L49

Falco, M., Hansen, S. H., Wojtak, R., et al. 2014, MNRAS, 442, 1887

Ftaclas, C., Struble, M. F., \& Fanelli, M. N. 1984, ApJ, 282, 19

Fujita, Y. 2004, PASJ, 56, 29

Guo, Q., Tempel, E., \& Libeskind, N. I. 2015, ApJ, 800, 112

Joachimi, B., Cacciato, M., Kitching, T. D., et al. 2015, SSRv, 193, 1

Karachentsev, I. D., \& Nasonova, O. G. 2010, MNRAS, 405, 1075

Karachentsev, I. D., Tully, R. B., Wu, P.-F., Shaya, E. J., \& Dolphin, A. E. 2014, ApJ, 782, 4

Kim, S., Rey, S.-C., Jerjen, H., et al. 2014, ApJS, 215, 22

Lee, J., Kim, S., \& Rey, S.-C. 2015a, ApJ, 815, 43

Lee, J., Kim, S., \& Rey, S.-C. 2015b, ApJ, 807, 122

Lee, J., Rey, S.-C., \& Kim, S. 2014, ApJ, 791, 15

Limousin, M., Morandi, A., Sereno, M., et al. 2013, SSRv, 177, 155

Mamon, G. A., Sanchis, T., Salvador-Solé, E., \& Solanes, J. M. 2004, A\&A, 414,445

Martínez, H. J., Muriel, H., \& Coenda, V. 2016, MNRAS, 455, 127

McLaughlin, D. E. 1999, ApJL, 512, L9

Mei, S., Blakeslee, J. P., Côté, P., et al. 2007, ApJ, 655, 144

Mould, J. R., Huchra, J. P., Freedman, W. L., et al. 2000, ApJ, 545, 547

Pak, M., Rey, S.-C., Lisker, T., et al. 2014, MNRAS, 445, 630

Paturel, G., Petit, C., Prugniel, P., et al. 2003, A\&A, 412, 45 
Pomarède, D., Tully, R. B., Hoffman, Y., \& Courtois, H. M. 2015, ApJ, 812,17

Porter, S. C., Raychaudhury, S., Pimbblet, K. A., \& Drinkwater, M. J. 2008, MNRAS, 388, 1152

Tully, R. B. 1982, ApJ, 257, 389 (T82)

Tully, R. B., Courtois, H., Hoffman, Y., \& Pomarède, D. 2014, Natur, 513, 71

Tully, R. B., Shaya, E. J., Karachentsev, I. D., et al. 2008, ApJ, 676, 184 (T08)
Tully, R. B., \& Trentham, N. 2008, AJ, 135, 1488

Vazza, F., Brüggen, M., Gheller, C., \& Wang, P. 2014, MNRAS, 445, 3706

West, M. J., \& Blakeslee, J. P. 2000, ApJL, 543, L27

Yoon, H., Chung, A., Sengupta, C., et al. 2015, PKAS, 30, 495

Yoon, J. H., Putman, M. E., Thom, C., Chen, H.-W., \& Bryan, G. L. 2012 ApJ, 754, 84 